\title{
Pharmacogenetic Analysis of Captopril Effects on Blood Pressure: Possible Role of the Ednrb (Endothelin Receptor Type B) Candidate Gene
}

\author{
J. ZICHA ${ }^{1}$, Z. DOBEŠOVÁ ${ }^{1}$, V. ZÍDEK ${ }^{1}$, J. ŠILHAVÝ ${ }^{1}$, M. ŠIMÁKOVÁ ${ }^{1}$, P. MLEJNEK ${ }^{1}$, \\ I. VANĚČKOVÁ ${ }^{1}$, J. KUNEŠ ${ }^{1}$, M. PRAVENEC ${ }^{1}$
}

${ }^{1}$ Institute of Physiology Academy of Sciences of the Czech Republic, Prague, Czech Republic

Received January 8, 2014

Accepted January 23, 2014

\section{Summary}

The objective of the current study was to search for genetic determinants associated with antihypertensive effects of angiotensin-converting enzyme (ACE) inhibitor captopril. Linkage and correlation analyses of captopril-induced effects on blood pressure (BP) with renal transcriptome were performed in the $\mathrm{BXH} / \mathrm{HXB}$ recombinant inbred (RI) strains derived from spontaneously hypertensive rat (SHR) and Brown Norway (BN-LX) progenitors. Variability of blood pressure lowering effects of captopril among RI strains was continuous suggesting a polygenic mode of inheritance. Linkage analysis of captoprilinduced BP effects revealed a significant quantitative trait locus (QTL) on chromosome 15. This QTL colocalized with cis regulated expression QTL (eQTL) for the Ednrb (endothelin receptor type B) gene in the kidney (SHR allele was associated with increased renal expression) and renal expression of Ednrb correlated with captopril-induced BP effects. These results suggest that blood pressure lowering effects of ACE inhibitor captopril may be modulated by the variants at the Ednrb locus.

\section{Key words}

Captopril - Blood pressure - Genetics • Ednrb gene • Spontaneously hypertensive rat

\section{Corresponding author}

M. Pravenec, Institute of Physiology Academy of Sciences of the Czech Republic, Vídeňská 1083, 14220 Prague 4, Czech Republic. E-mail: pravenec@biomed.cas.cz
Angiotensin-converting enzyme

(ACE)

inhibitors are widely used for the treatment of high blood pressure. ACE inhibitors do not lower blood pressure in all hypertensive patients (Dickerson et al. 1999, Struthers et al. 2001). Therefore it has been suggested that these differences in blood pressure lowering effects might be genetically determined. Identified responsible genetic determinants could serve as useful markers to predict the therapeutic efficacy of these drugs (Konoshita et al. 2011). The objective of the current study was to search for genetic determinants associated with antihypertensive effects of ACE inhibitor captopril in the $\mathrm{BXH} / \mathrm{HXB}$ recombinant inbred (RI) strains derived from spontaneously hypertensive rat (SHR) and Brown Norway (BN-Lx) rat. Figure 1A shows continuous variability of blood pressure lowering effects of captopril among RI strains which suggests a polygenic mode of inheritance. Linkage analysis of captopril-induced BP effects revealed a significant quantitative trait locus (QTL) on chromosome 15 (Fig. 1B). RI strains with BN-Lx genotype at the peak of QTL linkage exhibited significantly smaller captopril-induced BP reduction when compared with RI strains with SHR genotype $(-7.4 \pm 0.6$ vs. $-12.1 \pm 0.8 \mathrm{~mm} \mathrm{Hg}, \mathrm{P}=0.00003)$. This QTL is colocalized with cis regulated expresstion QTL (eQTL) for the Ednrb (endothelin receptor type $\mathrm{B}, \mathrm{ET}_{\mathrm{B}} \mathrm{R}$ ) candidate gene in the kidney (SHR allele was associated with increased renal expression when compared with BN-Lx allele) (Fig. 1B). In addition, we observed a significant correlation between renal expression of $E d n r b$ and captopril-induced BP effects (r=0.48, $\mathrm{P}=0.01)$. 
Sequence analysis of the BN-Lx and SHR Ednrb alleles revealed several polymorphisms in the promoter region, however, promoter analysis using luciferase reporter gene assay showed no differences in transcriptional activity of the Ednrb promoter region of the SHR strain when compared with the Ednrb promoter region of the BN-Lx strain (data not shown). This result suggests that cis regulated renal expression of Ednrb gene is determined by variants outside the tested promoter region. The Ednrb is a promising candidate gene for blood pressure lowering effects of captopril. Endothelin 1 (ET-1), a major regulator of vascular function acts through endothelin receptors type $A\left(E_{A} R\right)$ and type $B\left(E T_{B} R\right)$ that are coded for by Endra and Ednrb genes on rat chromosomes 19 and 15 , respectively. While $\mathrm{ET}_{\mathrm{A}} \mathrm{R}$ mediates ET-1induced vasoconstriction and has been implicated in the pathogensis of hypertension, the $\mathrm{ET}_{\mathrm{B}} \mathrm{R}$ induces the release of vasodilatation factors including nitric oxide or prostacyclin and has been also implicated in the clearance of ET-1 (Mazzuca et al. 2012). $\mathrm{ET}_{\mathrm{B}} \mathrm{R}$ plays an important role in sodium and water excretion in the kidney by reducing sodium reabsorption by inhibiting ET-1 action in the collecting ducts of the renal medulla (Kohan et al. 2011). The important role of $\mathrm{ET}_{\mathrm{B}} \mathrm{R}$ in regulation of sodium balance is supported by the fact that rats with mutant Ednrb gene are salt-sensitive (Gariepy et al. 2000) and that mice with collecting duct-specific targeting of Ednrb gene are hypertensive (Ge et al. 2006). Finally, it has been demonstrated that ACE inhibition increases expression of Ednrb in kidneys (Moridaira et al. 2003).

In summary, our study identified Ednrb as a candidate gene for captopril-induced blood pressure effects: (1) cis-eQTL for renal expression of Ednrb is located at the peak of QTL linkage for captopril-induced BP effects, (2) reduced renal expression of Ednrb in RI strains with SHR allele is associated with significantly smaller BP changes after captopril when compared to RI strains with the BN-Lx Ednrb allele, and (3) renal Ednrb expression correlated with captopril-induced BP effects. Taken together, these results provide compelling evidence that captopril effects might be mediated via affecting renal expression of $\mathrm{ET}_{\mathrm{B}} \mathrm{R}$.
A.

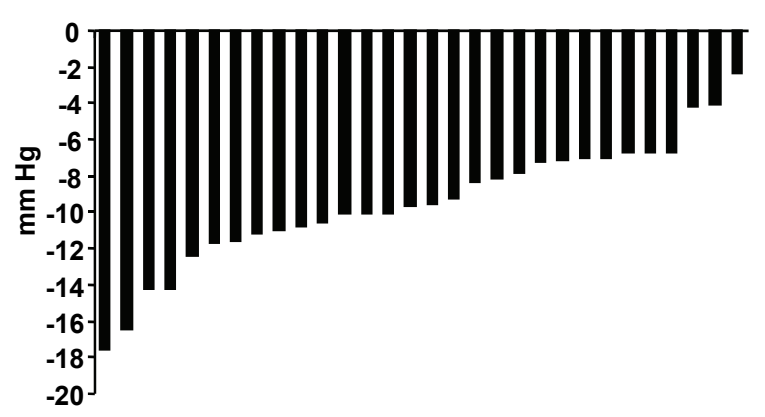

B.

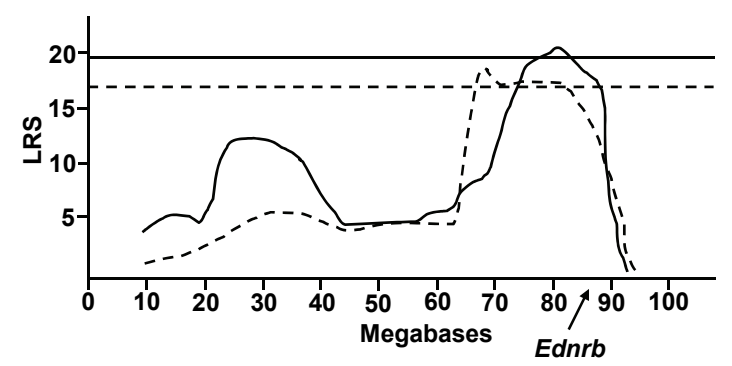

Fig. 1. Identification of genetic determinants of captopril-induced blood pressure effects in the BXH/HXB recombinant inbred (RI) strains. A. Distribution of captopril-induced BP effects among RI strains. Under light ether anesthesia, polyethylene catheters were inserted into the left carotid artery and jugular vein, and blood pressure was recorded in conscious animals after $24 \mathrm{~h}$ recovery. Basal mean arterial blood pressure values were monitored for $30 \mathrm{~min}$. Thereafter, an intravenous bolus of captopril was injected. B. Interval mapping of QTL associated with captopril BP effects (dashed line) colocalized with cis-regulated eQTL for renal expression of the Ednrb gene (solid line). Genome wide statistical significance for both QTLs (horizontal dashed and solid lines) was estimated by the permutation tests using the WebQTL online software (www.genenetwork.org).

\section{Conflict of Interest}

There is no conflict of interest.

\section{Acknowledgements}

This study was supported by grant LH11049 from the Ministry of Education, Youth and Sports of the Czech Republic to VZ.

\section{References}

DICKERSON JE, HINGORANI AD, ASHBY MJ, PALMER CR, BROWN MJ: Optimisation of antihypertensive treatment by crossover rotation of four major classes. Lancet 353: 2008-2013, 1999.

GARIEPY CE, OHUCHI T, WILLIAMS SC, RICHARDSON JA, YANAGISAWA M: Salt-sensitive hypertension in endothelin-B receptor-deficient rats. $J$ Clin Invest 105: 925-933, 2000. 
GE Y, BAGNALL A, STRICKLETT PK, STRAIT K, WEBB DJ, KOTELEVTSEV Y, KOHAN DE: Collecting ductspecific knockout of the endothelin B receptor causes hypertension and sodium retention. Am J Physiol Renal Physiol 291: F1274-F1280, 2006.

KOHAN DE, ROSSI NF, INSCHO EW, POLLOCK DM: Regulation of blood pressure and salt homeostasis by endothelin. Physiol Rev 91: 1-77, 2011.

KONOSHITA T, GENOMIC DISEASE OUTCOME CONSORTIUM (G-DOC) STUDY INVESTIGATORS: Do genetic variants of the renin-angiotensin system predict blood pressure response to renin-angiotensin systemblocking drugs? A systematic review of pharmacogenomics in the renin-angiotensin system. Curr Hypertens Rep 13: 356-361, 2011.

MAZZUCA MQ, KHALIL, RA: Vascular endothelin receptor type B: Structure, function and dysregulation in vascular disease. Biochem Pharmacol 84: 147-162, 2012.

MORIDAIRA K, MORRISSEY J, FITZGERALD M, GUO G, MCCRACKEN R, TOLLEY T, KLAHR S: ACE inhibition increases expression of the $\mathrm{ET}_{\mathrm{B}}$ receptor in kidneys of mice with unilateral obstruction. Am J Physiol Renal Physiol 284: F209-F217, 2003.

STRUTHERS AD, ANDERSON G, MACFADYEN RJ, FRASER C, MACDONALD TM: Nonadherence with ACE inhibitors is common and can be detected in clinical practice by routine serum ACE activity. Congest Heart Fail 7: 43-46, 2001. 